\title{
Philosophiques
}

Jean-Luc Marion, Réduction et donation. Recherches sur Husserl, Heidegger et la phénoménologie, Paris, P.U.F. (coll. " Épiméthée »), 1989, 312 pages.

\section{Claude Piché}

Volume 20, numéro 1, printemps 1993

URI : https://id.erudit.org/iderudit/027219ar

DOI : https://doi.org/10.7202/027219ar

Aller au sommaire du numéro

Éditeur(s)

Société de philosophie du Québec

ISSN

0316-2923 (imprimé)

1492-1391 (numérique)

Découvrir la revue

Citer ce compte rendu

Piché, C. (1993). Compte rendu de [Jean-Luc Marion, Réduction et donation. Recherches sur Husserl, Heidegger et la phénoménologie, Paris, P.U.F. (coll. «

Épiméthée »), 1989, 312 pages.] Philosophiques, 20(1), 222-224.

https://doi.org/10.7202/027219ar d'utilisation que vous pouvez consulter en ligne.

https://apropos.erudit.org/fr/usagers/politique-dutilisation/ 


\section{Jean-Luc Marion, Réduction et donation. Recherches sur Husserl, Heidegger et la phénoménologie, Paris, P.U.F. (coll. "Épiméthée "), 1989, 312 pages.}

\section{par Claude Piché}

Jean-Luc Marion se montre préoccupé dans cet ouvrage par la question de l'avenir de la phénoménologie. Il s'agit de savoir si la phénoménologie, par-delà ses réalisations effectives depuis ses tout débuts recèle encore un potentiel inexploité, qui demeure toutefois conforme à son essence. L'auteur voit dans l'approche phénoménologique non pas tant une tentative de réhabilitation, voire d'extension du champ de l'intuition, que l'affirmation d'une primauté accordée à la « donation du phénomène » (p. 53). Aussi la thèse du livre s'indique-t-elle d'emblée dans le titre: si la phénoménologie se caractérise par l'attention portée au surgissement du phénomène, l'ampleur et l'originarité de ce qui se donne seront directement proportionnelles à la radicalité de la réduction qui lui prépare la voie.

Pour développer cette thèse, l'auteur prend pour interlocuteurs privilégiés deux penseurs qui ont dégagé de la manière la plus vigoureuse le domaine de la phénoménologie : Husserl et Heidegger. La radicalité de l'attitude phénoménologique se traduit, selon la formule classique, par l'injonction à retourner aux choses elles-mèmes. Chez Husserl, la libération du regard rendue nécessaire par cette injonction prend la forme d'une réduction phénoménologique transcendantale qui fait apparaître l'ensemble des phénomènes comme autant d'objets pour la conscience. Non seulement Heidegger est-il quant à lui amené à stigmatiser un tel primat de l'interprétation objectivante de l'étant, mais à l'issue d'une réduction existentiale (puisqu'en vertu des Gundprobleme der Phänomenologie il y a bel et bien « réduction » chez Heidegger), il radicalise l'entreprise phénoménologique au point de faire de l'être le phénomène par excellence. A la faveur d'une réduction à et de l'appel (p. 304), l'auteur interviendra en fin de parcours pour remettre en question cette phénoménalité de l'être, et du même coup la conception heideggérienne de la destination foncièrement ontologique de la phénoménologie. Grâce à cette ultime réduction, la phénoménologie est susceptible de s'ouvrir à un champ de donation beaucoup plus large encore qu'insoupçonné - mieux : inoui. 
Réduction ct donation consiste pour l'essentiel en une discussion de textes de Husserl et de Heidegger qui présentent ici un intérêt stratégique. Le lecteur saura sans doute apprécier la qualité et la perspicacité du travail exégétique qui lui est offert. L'auteur sait faire un usage judicieux du corpus intégral de chacun des deux auteurs, sans négliger en outre les leçons de Heidegger publiées récemment dans la Gesamtausgabe. De même, les références à une importante littérature secondaire demeurent instructives et se font souvent critiques. L'ensemble de la démarche est subdivisé en six chapitres : I. La percee et l'élargissement ; II. L'étant et le phénomène: III. L'ego et le Dasein ; IV. Question de l'être ou différence ontologique; V. L'être et la région, et VI. Le rien et la revendication.

Parmi les nombreux enjeux soulevés, il convient sans doute d'attirer l'attention sur les chapitres IV et VI qui jouent un rôle de première importance pour la mise en place de la contribution propre de l'auteur. Celui-ci établit en toute évidence, et à l'encontre de l'opinion généralement reçue (p. 174), que Sein und Zeit (SZ) comporte déjà des occurrences de l'expression « différence ontologique » sans que l'on ait à attendre, du moins en ce qui a trait aux textes publiés, les écrits de 1928-ıg2g (p. I6g). notamment «Vom Wesen des Grundes ». Toutefois, et voilà qui est digne d'intérêt, la teneur de cette expression dans $S Z$ s'écarte de manière significative de celle des textes ultèrieurs, qui deviendra son acception « canonique » (p. I8I) : la différence clairement affirmée entre être et étant. En effet, dans $S Z$ l'expression marque non pas la différence entre l'être et l'étant, mais plutôt entre deux genres d'étants : le Dasein d'une part. et les autres étants qui n'ont pas le privilège de se questionner quant à leur être, d'autre part.

Cette variation sémantique repérée entre $S Z$ et les textes ultérieurs doit être interprétée selon l'auteur comme l'indice d'un déplacement théorique, luimême issu d'une difficulté interne à $S Z$. Si en 1927 il s'agit de porter l'être à la phénoménalité, cette opération commande un détour par le Dasein, par l'étant donc qui pose la question de l'être. Or l'auteur interprète l'inachèvement de SZ. et plus précisément la perplexité dont fait montre Heidegger dans ce finale qu'est le paragraphe 83 (p. 207), comme l'échec d'une phénoménologie ontologique dans laquelle vient s'interposer le Dasein à titre de médiation indispensable.

C'est en ce sens que l'expression « différence ontologique », telle qu'usitée après $S Z$, ne fera plus désormais référence qu'au face à face de l'être et de l'étant, sans prendre appui sur la question du sens de l'être ancrée dans une Analytique existentiale. D'où la valeur symptomatique de la conférence de 1929 « Was ist Metaphysik? », qui tout comme SZ fait référence à l'angoisse, mais cette fois pour tenter un accès au phénomène de l'être sans l'entremise de l'être-au-monde existential. Le chapitre VI présente une interprétation minutieuse de la conférence, de même que de sa «Postface » (1943) et de son «Introduction » (1949) qui constituent autant de relectures visant à confirmer avec toujours plus de force la présence latente, dans le texte original de la conférence, de la différence ontologique. Au terme de sa démarche, l'auteur, loin de se laisser convaincre du bien-fondé de l'accessibilité du phénomène d'être par la voie de l'angoisse et du rien, saisit un motif de la « Postface » qu'il propose d'exploiter pour sa structure formelle: l'« Anspruch des Seins ». Peut-être la 
voix de l'« être » n'est-elle pas le phénomène par excellence. Peut-être la donation qui revêt la forme de l'appel se situe-t-elle au-delà ou en deçà de l'être. Une chose est certaine à ses yeux : cette donation sui generis demeure indisponible pour une subjectivité transcendantale autarcique et inconditionnée (Husserl), ainsi que pour un Dasein qui s'approprie lui-même dans la résolution anticipatrice (Heidegger). Elle fait appel à l'homme à titre d'« interloqué ». ouvert à une revendication qui le surprend et le comprend.

Ce chapitre VI débouche sur la réévaluation de certains éléments qui, comme par exemple l'ennui et l'Anspruch, sont repris de Heidegger pour être dirigés cette fois contre lui. Ils conservent ici une allure encore programmatique, en sorte que l'auteur, dans ce livre, aura surtout insisté sur l'enracinement historique de son projet de renouvellement de la phénoménologie, laquelle est désormais susceptible d'adopter de nouveaux accents.

Département de philosophie

Université de Montréal 